\title{
Muir-Torre Syndrome Presenting as Sebaceous Adenocarcinoma and Invasive MSH6-Positive Colorectal Adenocarcinoma
}

\author{
Sunil Tulpule Hiyam Ibrahim Mohamed Osman Shoaib Zafar \\ Romana Kanta Gregory Shypula Mohammed A. Islam Shuvendu Sen \\ Abdalla Yousif \\ Department of Medicine, Raritan Bay Medical Center, Perth Amboy, N.J., USA
}

\section{Key Words}

Muir-Torre syndrome · Hereditary nonpolyposis colon cancer · Sebaceous carcinoma · MSH6

\begin{abstract}
Muir-Torre syndrome (MTS) is a rare genodermatosis, diagnosed by the presence of sebaceous neoplasms along with an internal malignancy, most commonly colorectal carcinomas. MTS is most commonly caused by microsatellite instabilities of the hMLH1 and hMSH2 mismatch repair genes, and is rarely caused by mutations of the hMSH6 gene. We describe the case of a 56-year-old male who presented with an enlarging mass on his back as well as hematochezia. The back mass was excised, and pathology confirmed microsatellite instability in MSH2 and MSH6. Abdominal CT and colonoscopy confirmed the presence of synchronous masses in the cecum, ascending colon, and the transverse colon. He refused any further workup or treatment, only to return 8 months later complaining of hematochezia and discomfort due to an enlarging mass protruding from the rectum. After consenting to surgical intervention, he agreed to outpatient chemotherapy treatment. The presence of sebaceous neoplasms should raise suspicion for the possibility of an associated internal malignancy.
\end{abstract}


Tulpule et al.: Muir-Torre Syndrome Presenting as Sebaceous Adenocarcinoma and Invasive MSH6-Positive Colorectal Adenocarcinoma

\section{Introduction}

Muir-Torre syndrome (MTS) is an autosomal genodermatosis considered to be a subtype of hereditary nonpolyposis colorectal cancer (HNPCC), and presents with a high incidence of various internal malignancies and sebaceous neoplasms. MTS is rare, with approximately 200 reported cases [1], more commonly affecting males, with a male-to-female ratio of 3:2 [2]. Sebaceous neoplasms present at a mean age of 53 years, and visceral malignancies are diagnosed at a mean age of 50 years [3]. The underlying genetic background of MTS is attributed to microsatellite instabilities due to germline mutations in DNA mismatch repair genes, most commonly hMSH2 and hMLH1, and less commonly hMSH6, which is responsible for base substitution mutations [4,5]. MSH6 mutations occur in $7.6 \%$ of HNPCC cases, but only in few reported cases of MTS. We present a case of MTS presenting as sebaceous adenocarcinoma of the mid-thoracic back with concurrent MSH6-positive, invasive colorectal cancer.

\section{Case Presentation}

A 56-year-old Ghanaian male with a medical history of hypertension presented to the emergency department complaining of fatigue, hematochezia, and back pain from a $7 \times 7 \times$ $4.2 \mathrm{~cm}$ exophytic, cauliflower-shaped, malodorous mass on his mid-thoracic back that increased in size over a period of 4 months (fig. 1a). The patient was anemic with a hemoglobin level of $7.0 \mathrm{~g} / \mathrm{dl}$, hyperglycemic with a serum glucose level of $261 \mathrm{mg} / \mathrm{dl}$, and stool guaiac positive. He lived alone, without family contacts, and was unaware of any family history of malignancy. The back mass was excised, and pathology revealed sebaceous carcinoma with free margins (fig. 1b). CT of the abdomen showed a 9-cm segment of mass-like thickening involving the ascending colon (fig. 2a), as well as irregular wall thickening and heterogeneity in the rectum (fig. 2b). Of note, CT also demonstrated $1.7-\mathrm{cm}$ adrenal nodules bilaterally. Colonoscopy with biopsy was performed, and pathology confirmed an invasive adenocarcinoma of the colon in the cecum arising from a tubular adenoma, carcinoma in situ in the ascending colon, and a tubulovillous adenoma in the transverse colon. All three tumors demonstrated microsatellite instability in MSH2 and MSH6 (fig. 3). The patient refused chemotherapy and colon resection, and was discharged after transfusion of 2 units of packed red blood cells and stabilization of his anemia. The patient was lost to follow-up.

Eight months later, he returned to the emergency department complaining of rectal bleeding for 2 days. He stated that he had had discomfort with defecation for 1 month and noticed a pink-colored mass protruding from his rectum that gradually increased in size. His hemoglobin level on readmission was $7.4 \mathrm{~g} / \mathrm{dl}$. The repeat CT results for the abdomen were relatively unchanged from those of his previous admission. The patient consented to total proctocolectomy. Intraoperatively, a careful exploration of the abdomen was manually performed and showed a large bulky tumor in the cecum, without evidence of any palpable nodal disease. Also, there was no evidence of any metastatic disease in the liver or in the omentum. A bulky rectal tumor was noted to be protruding through the anal canal and appeared to be involving the sphincter muscles. After proctocolectomy with ileostomy and omental flap, the patient recovered and agreed to outpatient chemotherapy treatment. 
Tulpule et al.: Muir-Torre Syndrome Presenting as Sebaceous Adenocarcinoma and Invasive MSH6-Positive Colorectal Adenocarcinoma

\section{Discussion}

MTS is diagnosed clinically, based on the presence of at least one sebaceous gland tumor associated with at least one internal malignancy [6]. The locations of skin lesions primarily observed in MTS are the head and neck, the eyelids, and the periocular region [5]. Sebaceous tumors precede visceral malignancies in $22 \%$, occur simultaneously in $6 \%$, and develop subsequently in $56 \%$ of the reported MTS cases [7-9]. The most common visceral malignancies occurring in MTS are colorectal and genitourinary. Therefore, some authors advocate genitourinary surveillance in addition to upper and lower gastrointestinal endoscopy for all patients with sebaceous tumors [10]. The presence of sebaceous skin lesions can support the earlier diagnosis of HNPCC in families [11]. Less commonly, the sebaceous tumors are associated with breast, hematologic, endometrial, and gastric malignancies. Previous studies have also suggested that screening for MTS should be carried out in all patients with sebaceous gland neoplasms [12]. Screening modalities may include radiological imaging, cytology, biopsies, or serum carcinoembryonic antigen levels. Abbas and Mahalingam [13] introduced a specific diagnostic algorithm based on the presence of microsatellite instabilities to identify patients with a propensity for this syndrome, as well as for the timely implementation of cancer prevention programs for patients and family members. Recent studies support the belief that mutational screening sometimes lacks sensitivity for MTS because not all mutations associated with this syndrome have been identified, and may therefore be just an additional diagnostic tool for the syndrome [6], which is diagnosed clinically.

In our case, microsatellite instability was found on MSH2 and MSH6. Although hMLH1 and hMSH2 are commonly associated with MTS, microsatellite instabilities of hMSH6 are rare. Our patient was evaluated for an internal malignancy likely because he presented with hematochezia and anemia, and coincidentally presented with a back mass which was found to be a sebaceous adenocarcinoma. However, because sebaceous neoplasms are rare in the general population, patients presenting with sebaceous tumors should be screened for visceral malignancy, including a review of systems and appropriate clinical investigations [14].

\section{Conclusion}

Whenever sebaceous tumors are identified, clinicians should consider the presence of internal malignancies. Detailed histories, particularly family histories, and physical examinations to identify masses should create a high index of suspicion, which can then be confirmed with pathology. Early recognition aids in the prompt treatment of patients, as well as screening of family members for early intervention.

\section{Statement of Ethics}

The authors declare that all examinations and interventions have been examined and approved by the appropriate Ethics Committee of Raritan Bay Medical Center and have therefore been performed in accordance with the ethical standards of the 1964 Declaration of Helsinki. 


\section{Case Reports in Oncology}

\begin{tabular}{|c|c|}
\hline \multicolumn{2}{|c|}{ Case Rep Oncol 2016;9:95-99 } \\
\hline DOI: $10.1159 / 000443788$ & $\begin{array}{l}\text { (C) } 2016 \text { The Author(s). Published by S. Karger AG, Basel } \\
\text { www.karger.com/cro }\end{array}$ \\
\hline
\end{tabular}

Tulpule et al.: Muir-Torre Syndrome Presenting as Sebaceous Adenocarcinoma and Invasive MSH6-Positive Colorectal Adenocarcinoma

\section{Disclosure Statement}

The authors declare no conflict of interest.

\section{References}

1 Hare HH, Mahendraker N, Sarwate S, Tangella K: Muir-Torre syndrome: a rare but important disorder. Cutis 2008;82:252-256.

2 Navi D, Wadhera A, Fung MA, Fazel N: Muir-Torre syndrome. Dermatol Online J 2006;12:4.

-3 Pettey AA, Walsh JS: Muir-Torre syndrome: a case report and review of the literature. Cutis 2005;75:149155.

4 Mercader P: Muir-Torre syndrome. Adv Exp Med Biol 2010;685:186-195.

5 Shalin SC, Lyle S, Calonje E, Lazar AJ: Sebaceous neoplasia and the Muir-Torre syndrome: important connections with clinical implications. Histopathology 2010;56:133-147.

6 Agiannidis C, Pana ZD, Molyva D, et al: Metachronous occurrence of colorectal cancer in a Muir-Torre syndrome patient presenting with recurrent sebaceous adenoma of the eyelid: case report and updated review of the literature. J Cutan Med Surg 2012;16:394-399.

7 Akhtar S, Oza KK, Khan SA, et al: Muir-Torre syndrome: a case report of a patient with concurrent jejunal and ureteral cancer and a review of the literature. J Am Acad Dermatol 1999;41(pt 1):681-686.

-8 Finan MC, Connolly SM: Sebaceous gland tumors and systemic disease: a clinicopathologic analysis. Medicine (Baltimore) 1984;63:232-242.

-9 Cohen PR, Kohn SR, Kurzrock R: Association of sebaceous gland tumors and internal malignancy: the MuirTorre syndrome. Am J Med 1991;90:606-613.

10 Ponti G, Longo C: Microsatellite instability and mismatch repair protein expression in sebaceous tumors, keratocanthoma, and basal cell carcinomas with sebaceous differentiation in Muir-Torre syndrome. J Am Acad Dermatol 2013;68:509-510.

-11 Tanyi M, Olasz J, Lukács G, et al: A new mutation in Muir-Torre syndrome associated with familial transmission of different gastrointestinal adenocarcinomas. Eur J Surg Oncol 2009;35:1128-1130.

$\$ 12$ Ingram JR, Griffiths AP, Roberts DL: All patients with sebaceous gland neoplasm should be screened for Muir-Torre syndrome. Clin Exp Dermatol 2009;34:264-266.

13 Abbas 0, Mahalingam M: Cutaneous sebaceous neoplasms as markers of Muir-Torre syndrome: a diagnostic algorithm. J Cutan Pathol 2009;36:613-619.

14 Bhaijee F, Brown AS: Muir-Torre syndrome. Arch Pathol Lab Med 2014;138:1685-1689.

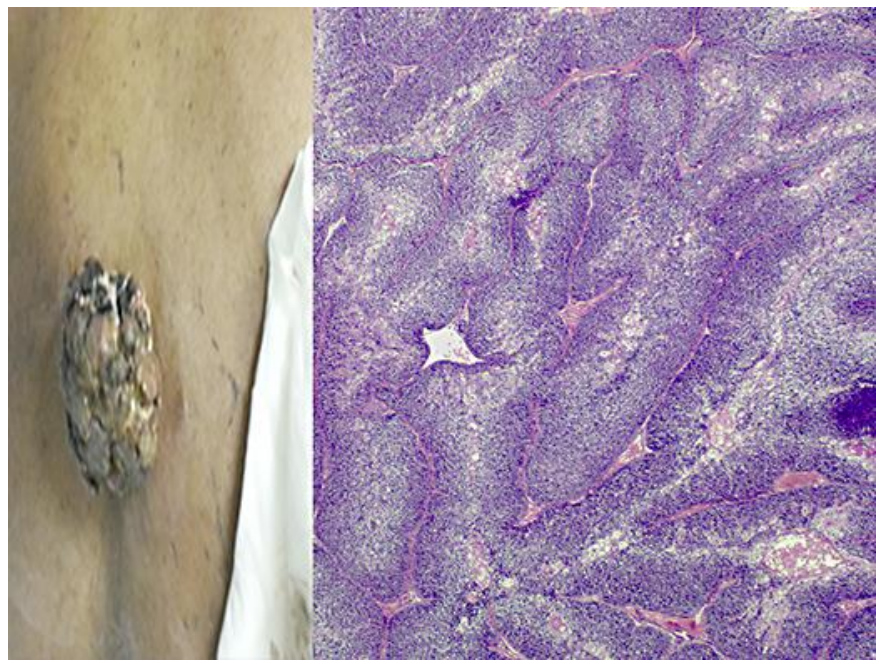

Fig. 1. Left: gross picture of the sebaceous carcinoma of the back. Right: histology confirming sebaceous carcinoma with free margins. 


\section{Case Reports in Oncology}

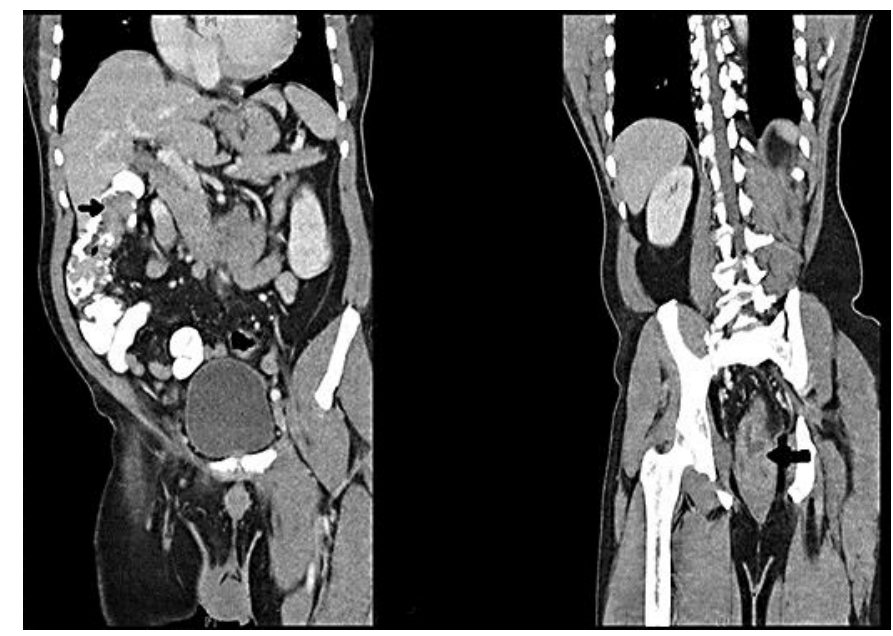

Fig. 2. Left: CT of the abdomen demonstrating a 9-cm mass in the ascending colon. Right: irregular wall thickening and heterogeneity in the rectum (arrow).

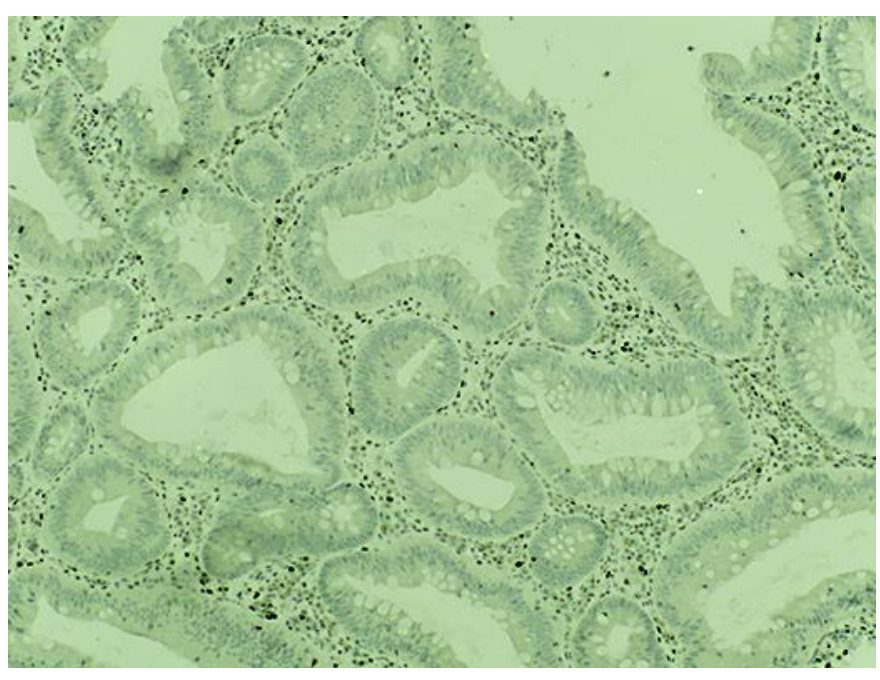

Fig. 3. Histology demonstrating microsatellite instability in MSH2 and MSH6.

Tulpule et al: Muir-Torre Syndrome Presenting as Sebaceous Adenocarcinoma and Invasive MSH6-Positive Colorectal Adenocarcinoma 